\title{
PERCEPCIÓN DE DOCENTES Y ESTUDIANTES SOBRE EL TRABAJO DE LA EDUCACIÓN EMOCIONAL EN EL AULA
}

\author{
David Hortigüela Alcalá \\ Vanesa Ausín Villaverde \\ Víctor Abella García \\ Vanesa Delgado Benito \\ Universidad de Burgos
}

\begin{abstract}
RESUMEN: La educación emocional se ha convertido en uno de los elementos pedagógicos fundamentales de cara a la mejora del desarrollo psicoevolutivo del estudiante. En esta investigación se realiza un análisis cualitativo con objeto de contrastar la percepción de estudiantes del Grado en Maestro de Educación Primaria, profesores universitarios de la citada titulación y maestros en activo en esta etapa obligatoria, acerca del rol que debe desempeñar la educación emocional en el aula. Los resultados muestran unanimidad en el alumnado a la hora de dar mayor peso a la educación emocional en las materias curriculares, algo que los docentes de los colegios reiteran, incluso afirmando que debería de ser una asignatura propia. Sin embargo, los docentes universitarios, en relación a la Formación Inicial del Profesorado (FIP), no están del todo de acuerdo con esta idea, debido en parte a la reducción de los contenidos instrumentales que podría conllevar. Se concluye que es necesario buscar acuerdos entre docentes de FIP y los de la etapa de primaria sobre cómo abordar este ámbito tan trascendental en la formación del individuo, ya que si los futuros docentes no han adquirido este bagaje durante su formación inicial universitaria difícilmente podrán aplicar estrategias en el día a día con sus estudiantes.
\end{abstract}

PALABRAS CLAVE: Educación emocional, percepción docente, percepción estudiante, formación inicial del profesorado, análisis cualitativo.

\section{PERCEPTION OF TEACHERS AND STUDENTS ON THE WORK OF EMOTIONAL EDUCATION IN THE CLASSROOM}

ABSTRACT: Emotional education has become one of the key educational elements for the enhancement of psychological and evolutionary development 
of student. In this research a qualitative analysis to contrast the perception of the students of the Degree in Primary, university professors of this degree and teachers of this stage on the role to be played by emotional education in the classroom is done. The results show how students reflect unanimously to give greater importance to the emotional education in curricular materials, something that teachers in schools reiterate, even claiming that there should be a separate subject. However, university teachers in initial teacher training (FIP) are not entirely agree with this idea, partly due to the reduction of instrumental content could lead. We conclude that it is necessary to seek agreement among teachers of FIP and primary stage on how to address this area as crucial in the formation of the individual, because if prospective teachers have not acquired this baggage during their initial training university can hardly apply on a day to day with their students.

KEYWORDS: Emotional education, teacher perception, student perception, initial teacher training, qualitative analysis.

Recibido: 08/03/2016

Aceptado: 20/07/2016

Correspondencia: David Hortigüela Alcalá, Universidad de Burgos, Facultad de Educación, Departamento de Ciencias de la Educación, C/Villadiego s/n, 09001 Burgos (España). Email: dhortiguela@ubu.es.

\section{INTRODUCCIÓN}

Diferentes estudios nacionales e internacionales (Decker y Roberts, 2015; FordConnors, Dougherty, Robertson y Paratore, 2015; López-Gómez, 2015) indican que, en la mayoría de los casos, la docencia suele enfocarse desde un planteamiento primordialmente estructurado sobre los conceptos y el ámbito cognitivo. Sin embargo, solamente se obtienen experiencias de éxito cuando el ámbito del conocimiento teórico se combina con los valores y las emociones, algo que lamentablemente todavía no tiene demasiado peso en los currículos educativos escolares (Winans, 2012).

Una de las ventajas más significativas que conlleva el trabajo de las emociones en el aula en las etapas de Educación Infantil y de Primaria es la mejora del ámbito social y conductual del niño (Cutri y Whiting, 2015), incrementándose además los valores de autoconcepto mediante el desarrollo de estrategias que fomenten la reflexión y las habilidades personales. Además, Habók (2015) indica que el trabajo emocional, asociado a planteamientos de carácter cooperativo, produce una mejora en la autoestima del alumno y favorece su relación con los demás, disminuyendo la conducta disruptiva y agresiva en el aula. Otras experiencias en el ámbito de la educación obligatoria y en programas formativos vinculados a la educación no formal, como la de Conderman y Hedin (2015), demuestran que el desarrollo de la 
gestión de las emociones mediante la reflexión y la indagación personal se asocia a la prevención del consumo de drogas y disminuye a medio plazo estados psicológicos de ansiedad y estrés.

Por lo tanto, parece lógico que la inteligencia cognitiva no sea la única que haya que tener en cuenta en el proceso formativo del alumno, ya que es la relación mente-cuerpo -que se encuentra implícita en la inteligencia emocional- la que permite desarrollar destrezas como la empatía, el conocimiento personal, las relaciones interpersonales y el desarrollo de competencias, dentro de una sociedad cada vez más cambiante y multicultural (Sucaromana, 2012). Además, el implemento de procesos formativos de educación emocional en conexión con la comunidad educativa se relaciona directamente con la obtención de una mayor felicidad a largo plazo por parte del estudiante (Yuan y Lee, 2015). Es por ello que se hace necesario reconfigurar un sistema educativo en el que prima, en mayor medida, el rendimiento académico del alumnado en base a estructuras de enseñanza individuales y cognitivas, más si cabe cuando queda demostrado que éste es solamente un factor de los muchos que influyen en el desempeño de competencias profesionales futuras (Mirzagitova y Akhmetov, 2015). En España, a pesar de ser un país con una escasa tradición respecto a la integración de la educación emocional en el sistema educativo, se han incorporado en los últimos años varios proyectos en colaboración con CASEL (Colaborative for Academic, Social and Emotional Learning). Sin embargo, como indica Mincu (2015), no podemos pretender que el aprendizaje social y emocional surja de manera espontánea y autónoma en el alumno, ya que debería enmarcarse bajo estructuras curriculares y educativas que sean controladas, respaldadas y consensuadas por los programas sociopolíticos instaurados.

A pesar de haberse demostrado que el campo de las emociones tiene cabida en diversos ámbitos: empresarial, político, de gestión..., es el educativo el que más trascendencia presenta en el desarrollo de la motivación del individuo (Thompson y Beymer, 2015). Esto supone un trabajo centrado en la reflexión, la escucha personal, la recapacitación, la valoración de las capacidades y la asunción de responsabilidades por parte del estudiante, lo que permite despertar el interés y la creatividad del alumnado hacia temáticas concretas a lo largo del proceso de enseñanza. Como indican Olsson y Gericke (2016), es preciso cambiar la concepción de que la educación es "café para todos", ya que esto genera que el discente no se decida, elija y se motive hacia ámbitos de conocimiento que le generen interés a largo plazo. ¿Es comprensible que una gran parte de los alumnos cuando acaban la educación obligatoria no tengan la menor idea de que estudios proseguir en la universidad?, y quizás lo más preocupante... ¿es qué todos tienen que ir a la universidad? Estudios con escolares (Ewing, Monsen y Kwoka, 2014) reflejan cómo el desarrollo emocional desde las primeras etapas se relaciona significativamente con el éxito social y laboral, no siendo ya tan trascendente el cúmulo de títulos y certificados académicos que se tengan. En definitiva, el rol del maestro no puede ceñirse únicamente a la transmisión de contenidos, ya que éstos se encuentran en internet en cuestión de segundos con solamente un clic. Es por ello esencial plantear propuestas que busquen la reflexión del niño, su autoncontrol emocional, la 
libertad de expresión, el compartir estados de ánimo, la búsqueda de soluciones grupales ante problemáticas surgidas, aprender a relajarse, a respirar, a sentir $y$, en definitiva, a ser felices (Zembylas, 2012).

Por lo tanto, parece que los profesores tienen que "deseducarse" en este sentido, olvidar los egos existentes en algunos casos y predicar con el ejemplo en lo que se refiere a aprender unos de otros. Para ello, la formación permanente es una herramienta fundamental a la hora de conexionar educación y sociedad, un binomio que todo profesor debiera tener en cuenta a través de un aprendizaje continuo. Así, y habiendo quedado de manifiesto la importancia que tiene la educación emocional, es necesario profundizar en la necesidad de coordinar este ámbito entre etapas, siendo imprescindible la formación inicial que los docentes universitarios den a los futuros maestros con el fin de que éstos, en un futuro cercano, lleven a cabo modelos pedagógicos similares.

En esta línea, el aporte relevante de esta investigación consiste en analizar, dando voz a los principales "actores" implicados en el proceso, cómo se forma a los futuros maestros en educación emocional, cuáles son las estrategias didácticas, metodológicas y evaluativas empleadas en el ámbito universitario y cómo esto repercute cuando los estudiantes realizan sus prácticas en los centros escolares. Este proceso secuencial, progresivo y deductivo, arroja datos que permiten comprender la situación actual y los porqués de un tipo de actuaciones u otras, algo que contribuye significativamente a la bibliografía existente sobre la temática. Por tanto, los objetivos del estudio son: a) Contrastar la percepción que tienen los docentes universitarios, los de la etapa de primaria y los estudiantes del Grado en Maestro de Educación Primaria sobre el rol que ha de desempeñar la educación emocional en el aula y b) Analizar en qué medida desde la formación inicial del profesorado se trabajan actividades vinculadas al ámbito emocional, la repercusión que tiene ésta en las primeras etapas y el modo en el que habría que enfocar el trabajo para que repercutiera positivamente en la sociedad.

\section{Método}

\section{Participantes}

Han participado 23 alumnos (13 mujeres y 10 hombres) matriculados en cuarto curso del Grado de Maestro de Educación Primaria en la Facultad de Educación de la Universidad de Burgos. Tienen una media de edad de $20.43 \pm 2.49$ años. También participaron ocho docentes universitarios (cuatro mujeres y cuatro hombres) que imparten docencia en esta titulación. Todos ellos pertenecen a diversas áreas de conocimiento, tienen una media de edad de $43.22 \pm 7.83$ años y $11.24 \pm 6.75$ años de experiencia en el ámbito universitario. Uno tiene la categoría de Catedrático de Escuela Universitaria, dos de ellos de Titular de Universidad, uno de Contratado Doctor, dos de Ayudante Doctor y los otros dos de Asociado 6+6.

Además, formaron parte del presente estudio siete maestros de la etapa de primaria de la ciudad de Burgos (cuatro mujeres y tres hombres) que han tutorizado el Prácticum I y/o II a estudiantes de la UBU de esta titulación. Tienen una media de edad de $47.38 \pm 9.62$ años y $14.31 \pm 11.38$ años de experiencia. Cuatro de ellos 
son funcionarios de carrera con destino definitivo, uno con destino en expectativa, uno interino y otro trabaja en un colegio concertado. Todos los participantes de la investigación fueron seleccionados por criterios de accesibilidad y representatividad en formación relativa a la etapa de primaria.

\section{Instrumento}

Se ha empleado el grupo de discusión estructurado como instrumento de recogida de la información para cada uno de los grupos de participantes (estudiantes, docentes universitarios y maestros de primaria). Este procedimiento, habitual en la metodología cualitativa, permite el diálogo, la participación y la obtención de la información desde una perspectiva crítica y contrastada (Sönmez, 2015).

La presencialidad en su puesta en práctica y el carácter vivencial y experimental sobre el que los participantes argumentan sus respuestas favorece el debate y la riqueza de la información obtenida, apoyándose en su experiencia y en la repercusión que tiene el contenido en su ámbito formativo y profesional (Mosha, 2015). Por ello, el enfoque utilizado es el de la entrevista grupal abierta, lo que permite la saturación de la información y el contraste de percepciones (Daghan y Akkoyunlu, 2014). Se otorga un enfoque multidisciplinar al instrumento, ya que los docentes universitarios, además de pertenecer a diferentes áreas de conocimiento, imparten docencia en una etapa no obligatoria, algo que no sucede con los maestros de primaria. Cada grupo de discusión es grabado en vídeo, lo que permite mayor facilidad en la posterior transcripción de los datos.

En cada uno de los tres grupos de discusión se emplea un guion estructurado con una serie de cuestiones vinculadas a las creencias y experiencias vivenciadas en torno a la educación emocional. Se realizan seis en cada una, siendo éstas adaptadas al rol y a las características de los grupos participantes. En la tabla 1 se reflejan las cuestiones empleadas con el grupo de los alumnos.

Tabla 1. Guión básico utilizado para el grupo de discusión final con los alumnos

1. ¿Consideráis que la educación emocional es importante dentro de las asignaturas universitarias? ¿Por qué?

2. ¿Os han formado lo suficiente en educación emocional?

3. ¿Trabajaréis el día de mañana estrategias de aula para fomentar el campo de las emociones?

4. ¿Qué puede aportar la educación emocional en el niño de primaria?

5. ¿Cómo se puede relacionar lo cognitivo con lo emocional? ¿lo vivenciasteis como alumnos en primaria? ¿y en la universidad?

6. ¿Qué competencias fundamentales en la vida creéis que pueden ser adquiridas a través del trabajo de las emociones en el aula?

En la tabla 2 se presenta las preguntas trabajadas en el grupo de discusión llevado a cabo con los docentes universitarios. 
Tabla 2. Guión básico utilizado para el grupo de discusión final con los docentes universitarios

1. ¿Incorporáis el trabajo de las emociones en vuestra docencia universitaria? ¿Por qué sí o por qué no?

2. ¿Asistís a seminarios, jornadas o revisáis información relativa al ámbito de las emociones en la escuela?

3. ¿Por qué consideráis que actualmente se da tanta relevancia a este campo cuando antes no se hacía?

4. ¿Qué puede aportar la educación emocional a los futuros maestros/as de primaria?

5. ¿Consideráis que el trabajo de las emociones puede potenciar el ámbito cognitivo o que sin embargo se disminuye? ¿Por qué?

6. ¿En qué medida puede influir el hecho de que el futuro docente haya recibido esta formación para su práctica docente?

En la tabla 3 se observan la cuestiones que se preguntaron a los maestros de primaria.

Tabla 3. Guión básico utilizado para el grupo

de discusión final con los maestros de primaria

1. Incorporáis el trabajo de las emociones en vuestras clases de primaria? ¿Por qué sí o por qué no?

2. ¿Os formáis fuera de la escuela en relación al ámbito de las emociones?

3. ¿En vuestro centro educativo se trabaja la educación emocional? ¿Este trabajo es a nivel de centro o de profesores individualmente?

4. ¿Qué puede aportar la educación emocional al desarrollo educativo del niño?

5. ¿Está reñido el trabajo de las emociones con el aprendizaje y/o el rendimiento académico?

6. ¿Cuáles son las mejoras y ventajas que conlleva el trabajo emocional en la etapa de primaria?

\section{Diseño y procedimiento}

Se emplearon tres grupos de discusión diferentes en función de los participantes de la investigación. Todos ellos se llevaron a cabo en un periodo comprendido de tres semanas, habiéndose estructurado el proceso en las siguientes fases:

- Fase 1. Diseño del estudio y contacto con participantes: tras delimitarse los objetivos del estudio, diseñar el tipo de metodología a llevar a cabo y estructurar las cuestiones que integran cada uno de los grupos de discusión, se procedió a contactar con los participantes. Los 23 estudiantes pertenecen a un grupo práctico de una asignatura del cuarto curso del Grado en Primaria que imparte uno de los investigadores, por lo que se les comentó la posibilidad de participar en la investigación en una de las clases. A los ocho docentes que imparten en la titulación se les comentó personalmente la posibilidad de participar en la investigación, debido a la fácil accesibilidad que tenían los investigadores por ser compañeros de Departamento y Facultad. Finalmente, 
se contactó vía email con los siete maestros. Se les dió a conocer las pretensiones de la investigación con el fin de saber si estaban dispuestos a colaborar en la misma y así fijar la fecha de la realización del grupo de discusión. Todos ellos mostraron una alta motivación hacia la temática del estudio.

- Fase 2. Puesta en práctica de los grupos de discusión y análisis de la información: todos los grupos de discusión se llevaron a cabo en la Facultad de Educación de la Universidad de Burgos. Con los alumnos se aprovechó una de las clases para Ilevarlo a cabo. Con los docentes del Grado se utilizó el seminario del Departamento para su realización y con los maestros se reservó un aula, aprovechando la reunión establecida para asignatura del prácticum. Los tres grupos de discusión tuvieron una duración de dos horas y todos ellos fueron grabados en vídeo para la posterior transcripción de los datos. Tras su recapitulación se procedió a la generación de categorías y su análisis.

- Fase 3. Revisión del procedimiento, discusión de los datos y establecimiento de líneas de trabajo futuras: se constató que las subcategorías aportaban a las categorías principales, se trianguló y saturó la información y se generaron las discusiones en función del aporte del estudio y de los objetivos planteados.

Desde el principio de la investigación se aseguró a los participantes su confidencialidad en las respuestas y el anonimato, garantizando que únicamente se utilizarían los resultados con fines científicos. Además se les comunicó que los resultados obtenidos eran abiertos, dándoles la posibilidad de compartir los mismos una vez que estos fueran obtenidos.

\section{Análisis empleado}

La metodología empleada en la investigación es de carácter cualitativo, ya que profundiza en las valoraciones y percepciones de los sujetos participantes con el fin de reflexionar sobre los procesos educativos vivenciados y experimentados (Petersen, 2012). Se incide en el contraste de valoraciones que realizan los agentes implicados en la etapa de primaria (maestros, futuros maestros y docentes universitarios) sobre el rol de la educación emocional. Este contraste entre profesionales educativos permite potenciar las interacciones existentes a través de análisis fenomenológicos e interpretativos (Caesar, 2012).

Toda la información recogida en los grupos de discusión fue triangulada y saturada a partir de la generación de categorías y subcategorías derivadas de la codificación de los extractos de texto más significativos (Saldaña, 2009). La primera extracción de resultados fue revisada sistemáticamente por los investigadores del estudio, realizando un análisis crítico e independiente. Toda la información recogida en las categorías fue analizada bajo los criteros de articulación y fragmentación de la "Grounded Theory" (Strauss y Corbin, 2002). Debido al gran volumen de información obtenido se utilizó la estructura de la categorización emergente, atendiendo para ello a una codificación axial, abierta y selectiva. Es por ello que el apartado de resultados se presenta a partir de la contabilización de extractos de texto obtenidos en cada categoría.

Es preciso destacar que la retroalimentación y el contacto continuo entre investigadores y participantes permitió un conocimiento real y profundo tanto de los datos como de la manera en la que éstos fueron obtenidos. 


\section{Generación de categorías y su categorización}

Como se ha comentado anteriormente, se crearon tres categorías comunes para los datos obtenidos en los grupos de discusión realizados, respondiendo de este modo a los objetivos del estudio. Todos los datos fueron analizados por el programa de computación cualitativo WEFT QDA. Estas categorías son:

- Nivel de trabajo del ámbito emocional en el aula: se registra todo lo relacionado con las propuestas de intervención desarrolladas para el trabajo de las emociones en los niños, analizando el grado de implantación de estrategias pedagógicas en las materias de la etapa.

- Estrategias de actuación de la educación emocional en la formación del individuo: se analizan las respuestas relativas a la relevancia y trascendencia que puede tener el trabajo emocional en el desarrollo psicoevolutivo del niño, contemplando cómo aplicar enfoques metodológicos compatibles con el tratamiento curricular.

- Relación entre rendimiento académico y educación emocional: se incluye la información vinculada con la variable de calificaciones en las materias y los criterios de evaluación utilizados para la superación de cada uno de los cursos.

El ajuste de la información a cada categoría favorece una mayor especificación de la misma y, como consecuencia, un análisis más exhaustivo, ajustado y comprensivo de los datos (Lee, Mishna y Brennenstuhl, 2010).

\section{Codificación de los extractos de texto en resultados}

Para la mejor interpretación de los datos se han utilizado diferentes acrónimos, asignado uno a cada grupo de discusión. Para el grupo de discusión de los estudiantes se empleó (GDE), para el de los docentes universitarios (GDU) y para el de los maestros (GDM).

\section{Resultados}

Toda la información extraída en los grupos de discusión se presenta de manera específica en cada una de las categorías del estudio. A través del análisis de patrones cruzados se presentan el número de extractos literales de texto resultantes, mostrándose aquellos más significativos y coincidentes.

\section{Nivel de trabajo del ámbito emocional en el aula (214 extractos de texto)}

Se observa cómo los alumnos del Grado en Maestro de Educación en Primaria exponen que a lo largo de la titulación no se ha trabajado mucho el ámbito emocional, manifestado que la mayoría de la docencia se enfoca hacia los contenidos específicos de cada asignatura:

"Al final cada asignatura, salvo excepciones, se centra específicamente en los contenidos específicos, estrategias basadas en educación emocional no hemos vivenciado muchas $[\ldots]$ ". "Se supone que todos aquí estamos para ser maestros, si está demos- 
trado que la educación emocional es positiva para el desarrollo de niño, ¿por qué no se potencia esto en la carrera?". "Es algo que se está trabajando en los colegios, y la universidad debería de trabajarlo paralelamente [...]" (GDE).

La percepción de los docentes universitarios participantes difiere, en parte, de la de los estudiantes, argumentado que no puede pretenderse que los contenidos de las guías docentes queden mermados por trabajar contenidos de carácter transversal más vinculados al desarrollo psicoevolutivo del niño. Sin embargo, otros docentes son más autocríticos con el tema:

"Esto va por modas, cada año es una cosa: aprendizaje por proyectos, comunidades de aprendizaje, bilingüismo...¿itenemos entonces que modificar nuestras asignaturas todos los años?[...]". "Creo que nuestra responsabilidad es que enseñemos bien nuestras asignaturas, que para eso somos expertos, [...], suficiente tarea es ya esa". "En cierta manera os comprendo pero discrepo [...], nuestra responsabilidad también es adaptarnos a los contextos actuales, formamos a futuros docentes, por lo que creo que la educación emocional ha de estar siempre presente" (GDU).

Sin embargo, los maestros de la etapa son más categóricos en esto, garantizando que independientemente de la asignatura si no se trabajan las emociones en los niños dificilmente se puede educar y enseñar algo. Afirman también la necesidad de que los maestros en activo se formen en este aspecto:

“¿Cómo va a aprender un alumno si no sabe controlar y gestionar sus emociones? [...], aunque la pregunta sería, ¿sómos nosotros capaces de hacerlo?" "De hecho, podría ser una asignatura propia, ya que está demostrado que lo importante no es el aprendizaje cognitivo, sino el relacional, el empático [...]". "Hace unos años primaba tu rendimiento académico, tu capacidad de memorización...esto actualmente es solamente un complemento que ha de estar unido a las habilidades los sociales y otro tipo de inteligencias [...] ¿se tiene en cuenta esto en la formación de los futuros maestros?" (GDM).

\section{Estrategias de actuación de educación emocional en la formación del individuo}

(231 extractos de texto)

Existe una mezcolanza de ideas entre los alumnos participantes acerca del tipo de trabajos que se pueden Ilevar a cabo en el aula para desarrollar las emociones:

"Hay que saber escuchar, hacer que el niño exprese sus emociones [...], para ello es necesario diseñar actividades que favorezcan la relación con los demás y traten el tema de los valores". "El tema de las emociones es importante, pero creo que también depende de cada niño [...], no sé hasta que punto debemos invertir tiempo en este aspecto" (GDE).

Los docentes universitarios abogan por un tratamiento más de acompañamiento y transversal que por un enfoque más específico implantado en las materias. Además otorgan más está responsabilidad a los maestros de la etapa que a las enseñanzas no obligatorias:

"Independientemente de que podamos estar más o menos de acuerdo con el papel que ha de desempeñar la educación emocional en la escuela, considero que el 
tratamiento que se haga en clase ha de estar vinculado con determinados contenidos o materias [...]". "Son los maestros de primaria los que más tienen que incidir en esto, nosotros desde la universidad tenemos que potenciar diversidad de contenidos de forma más consistente" (GDU).

Los maestros de Primaria no comparten esta idea tan fragmentada y abogan por dirigir el paradigma educativo de las primeras etapas hacia los valores y las emociones. Además recalcan la importancia de trabajarlo en la enseñanza universitaria:

"Los aspectos cognitivos carecen de toda importancia si no se asocian a un trabajo emocional y de valores sólido [...]". "Es importante que desde las primeras etapas aprendan a entenderse, escuchar a los demás, ser críticos, autoevaluarse, buscar soluciones ante los problemas y ser feliz por y para los demás [...], algo que debe profundizarse en las enseñanzas universitarias". "Esto es aplicable a todas las áreas y para ello es necesario que los maestros nóveles que se incorporan a las escuelas tengan esto muy presente" (GDM).

Relación entre rendimiento académico y educación emocional (229 extractos de texto)

La experiencia de los estudiantes en la titulación les hace pensar que su calificación en las asignaturas cursadas ha dependido básicamente de aspectos cognitivos y de su capacidad de memorización. También reconocen la disparidad de criterios empleados entre unos docentes y otros a la hora de valorar esto:

"La mayoría de los profesores te piden que memorices, expongas trabajos.......], al final casi toda la nota depende de la realización de una prueba individual". "Hemos tenido profesores que evalúan de otra manera, demostrando que trabajar habilidades sociales o temas más relacionados con los valores de la escuela no quiere decir que se aprenda menos" (GDE).

Los profesores universitarios reconocen la dificultad existente a la hora de relacionar lo más puramente académico con el plano de las emociones, desvinculando en cierto modo lo que un futuro maestro debe saber a nivel conceptual con la actitud con la que puede afrontar su docencia:

"Es complicado integrar las emociones cuando tienes que enseñar algunos contenidos [...], de hecho parece difícil pronosticar que un futuro docente aborde el campo de las emociones en clase simplemente por una forma $u$ otra en la que la asignatura se haya presentado". "Si la docencia es vocacional, este ámbito de las emociones ha de ir en la propia persona, pero lo que hay que enseñar en una asignatura no ha de cambiarse, más que nada por el poco tiempo que tenemos" (GDU).

Los maestros de primaria divergen de este planteamiento al relacionar el aprendizaje emocional con el curricular, reflejando que la adquisición de contenidos no sirve de nada si no pueden ser aplicados a contextos reales en los que las emociones juegan un rol importante:

"Sabemos que hay que trabajar la creatividad, el desarrollo de la autonomía y la motivación desde las primeras etapas [...], las emociones tienen un papel fundamental en que esto sea posible ya que permiten que el alumnado se conozca más y entienda 
mejor lo que le rodea". "¿De qué sirve ser brillante académicamente si luego no eres capaz de desenvolverte en la sociedad de manera óptima? [...], creo que debemos de ir más allá de las propias actividades de los libros de texto" (GDM).

\section{Discusión}

Se ha observado que en las tres categorías del estudio los grupos de participantes difieren en relación al cómo se está trabajando actualmente la educación emocional en las aulas, cuál es el rol que ha de desempeñar en el proceso de enseñanza y aprendizaje del niño y de los futuros maestros y cómo puede vincularse al rendimiento académico del estudiante. En este sentido, los maestros de la etapa de primaria abogan por un tratamiento integrado de la educación emocional en los contenidos más curriculares, algo en lo que coinciden los estudiantes en función de sus escasas experiencias a lo largo de la carrera. Entre los docentes universitarios de FIP existe más controversia, debido en parte a la importancia y a la especificidad de los contenidos de cada asignatura.

En relación a la primera categoría, relativa al nivel de trabajo del ámbito emocional en el aula, los estudiantes valoran como escasa la formación que han recibido en la universidad al respecto, algo que no ven adecuado al considerarlo relevante en sus competencias profesionales futuras. En este sentido, otras experiencias como las de Brown (2015) reflejan una mayor satisfacción de los maestros nóveles hacia su trabajo cuando han recibido formación relativa al ámbito de la empatía y las relaciones interpersonales. La valoración que han realizado los docentes universitarios es dispar en este sentido, ya que algunos consideran que los contenidos de las asignaturas pueden disminuirse si se trabaja el ámbito emocional, mientras otros creen que esto no es tan grave si verdaderamente es útil para la formación del futuro maestro. O'Sullivan (2011) indica que es fundamental que los programas de las enseñanzas universitarias docentes sean revisadas no únicamente de manera superficial y curricular, sino en función de la estructura de los contenidos y su transferibilidad a los contextos reales. Por su parte, los maestros de primaria de la presente investigación relacionan las emociones con la educación y el querer aprender, algo en lo que necesariamente tienen que ser formados los maestros. En esta línea, es imprescindible el rol que ha de desempeñar la formación permanente del docente, clave fundamental del desarrollo social (Barrett, Gross, Christensen y Benvenuto, 2001; Hortigüela, Pérez-Pueyo y Fernández Río, 2016).

Respecto a la segunda categoría del estudio, referente a las estrategias de actuación de educación emocional en la formación del individuo, los alumnos presentan disparidad de opiniones acerca de cómo afrontar este tema transversal en el aula, valorando para ello las características del niño. Algunos estudios como el de Bennett (2014) indican que actualmente los docentes nóveles tienen una mayor conciencia de la importancia que tiene trabajar las emociones en las primeras edades, pero esto no se relaciona en algunos casos con estrategias concretas de actuación que provoquen mejoras. Por su parte, los docentes universitarios han reflejado cómo estos aspectos más vinculados al ámbito personal del alumnado no les corresponde a ellos trabajarlos en el aula, sino que es más lógico que lo hagan los maestros de escuela. Sin embargo, los maestros participantes no están de acuerdo, argumentando que si 
no son los maestros jóvenes los que abordan las emociones en el aula es verdaderamente complicado que se consoliden propuestas más estables a largo plazo. Parece, por lo tanto, que uno de los aspectos más relevantes para generar cambios en los procesos educativos es la linealidad y la coordinación que tiene que existir entre todos los responsables de la formación en la diversidad de las etapas (Dolan, 2012).

La tercera categoría de relación entre rendimiento académico y educación emocional ha presentado disparidad de criterios en el estudio. Los estudiantes indican que en la mayoría de los casos su rendimiento académico se vincula a la nota individual que se alcance en un prueba o examen basada en la memorización, aunque destacan que otros docentes emplean métodos evaluativos más enfocados a otro tipo de aprendizaje. Como indican López-Pastor y Palacios (2013), los enfoques tradicionales de enseñanza siguen siendo los más habituales en la docencia universitaria, aunque cada vez existen más profesores eclécticos que los combinan con procesos de evaluación formativa. Los docentes de FIP establecen que los contenidos tienen que ser desarrollados de un modo o de otro, pero su adquisición es fundamental en un periodo de tiempo cada vez menor. De nuevo, los maestros de la etapa plantean una perspectiva diferente, abogando por la vinculación del plano más emocional a la adquisición de contenidos, ya que la mera adquisición de éstos de manera aislada no garantizan su aplicación. Estudios recientes como el de Batra (2013) reflejan que las personas que vivenciaron una educación en la que se potenciaron sus emociones reconocen 15 años más tarde ser más felices en su día a día que aquellos que no lo hicieron. Además, Flecha y Villarejo (2015) indican que el aprendizaje es más sólido cuando ha sido sustentado en tres pilares: cognitivos, valores y emociones, afirmando que un hecho es tan recordado como de importante fuera el contexto en el que se tuvo la experiencia. Esto se asocia con las competencias que deberán adquirir los estudiantes a lo largo de su proceso de enseñanza, no siendo tan importante el qué digo que sé o de lo que soy capaz, como el hecho de poder demostrarlo fehacientemente (Zlatkin-Troitschanskaia, Blömeke y Pant, 2015).

\section{Conclusiones}

En este apartado se dará respuesta a cada uno de los objetivos de la investigación, comentando cuál es el principal aporte del estudio, sus limitaciones, así como las futuras líneas de investigación para su complementación y mejora. También se destaca para qué colectivos puede ser de interés.

En relación al primer objetivo del estudio, se ha comprobado cómo la percepción de los agentes implicados en la educación emocional del niño de primaria es bastante dispar, siendo los maestros de la etapa los que abogan por un tratamiento específico dentro de las materias escolares, destacando la importancia que tiene que los futuros maestros adquieran conocimiento sobre esta temática. Sin embargo, los docentes universitarios no le otorgan tanto peso, argumentado en la mayoría de los casos que la enseñanza universitaria ha de centrarse en aspectos más propios de los conocimientos específicos reflejados en las guías docentes de cada una de las materias. Por su parte los alumnos han manifestado la carencia de estrategias emocionales que han recibido a lo largo de su formación, salvo en asignaturas concretas en las que la evaluación iba más allá que la memorización de contenidos asociados a pruebas de calificación finales e individuales. 
Respecto al segundo objetivo, los tres grupos de discusión participantes coinciden en la relevancia que tiene saber gestionar tus emociones de cara a un desarrollo de competencias sociales óptimas, no encontrando un punto de acuerdo a la hora de definir quiénes tienen que ser los responsables de su implantación y desarrollo y a través de que programas curriculares o socioculturales han de generarse estos procesos. Por tanto, se observa una discordancia en relación al cómo han de comenzar a implantarse estos enfoques pedagógicos: a) si a partir de la escuela o b) desde la formación inicial del profesorado.

El principal aporte del estudio ha sido dar voz, de manera estructurada y categorizada, a los principales agentes implicados en el desarrollo de la etapa de primaria acerca de un tema tan trascendental como es la educación emocional. Para ello se ha desarroIlado una metodología cualitativa, utilizando grupos de discusión con futuros maestros, maestros ejercientes y docentes del Grado en Educación Primaria, que han arrojado datos que contribuyen a la bibliografía existente en nuestro país sobre la temática.

El artículo presenta algunas limitaciones. En primer lugar aborda las etapas de primaria y universitaria, por lo que sería interesante contrastar los datos con otros periodos educativos como el de infantil y secundaria, comprobando en qué medida la edad y el desarrollo psicoevolutivo del estudiante influye en el desarrollo de sus emociones. En segundo lugar, y debido al enfoque cualitativo empleado, el número de los participantes no es muy elevado, por lo que podrían combinarse los datos obtenidos con metodologías más cuantitativas o de carácter mixto.

Consideramos que el artículo puede ser de interés para todos aquellos docentes, de cualquier etapa educativa, interesados en implantar modelos pedagógicos vinculados a la educación emocional. También para los coordinadores y/o responsables de la elaboración de programas académicos y curriculares, ya que podrán reflexionar sobre la importancia que tiene desarrollar el ámbito de las emociones en el aula.

Por lo tanto, parece de especial relevancia que desde las administraciones educativas se implanten procesos curriculares y formativos que garanticen la necesaria coordinación de los diferentes centros y docentes a la hora de abordar un tema tan transversal, relevante y competencial.

\section{REFERENCIAS BIBLIOGRÁFICAS}

Barrett, L. F., Gross, J. J., Christensen, T. C. y Benvenuto, M. (2001). Knowing what you're feeling and knowing what to do about it: Mapping the relation between emotion differentiation and emotion regulation. Cognition and Emotion, 16, 713-724.

Batra, S. (2013). The Psychosocial Development of Children: Implications for Education and Society--Erik Erikson in Context. Contemporary Education Dialogue, 10(2), 249-278. DOI: http://doi.org/10.1177/0973184913485014.

Bennett, L. (2014). Putting in More: Emotional Work in Adopting Online Tools in Teaching and Learning Practices. Teaching in Higher Education, 19(8), 919-930. DOI: http://doi.org/10.1080/13562517.2014.934343.

Brown, C. P. (2015). Taking and Teaching the Test Are Not the Same: A Case Study of FirstYear Teachers' Experiences in High-Stakes Contexts. Teachers and Teaching: Theory and Practice, 21(8), 1026-1044. DOl: http://doi.org/10.1080/13540602.2015.1005870. 
Caesar, C. (2012). The Relevance and Appropriateness of Positivist and Interpretative Approaches for Exploring Quality Education in Schools in St. Lucia, a Small Caribbean State. Educational Research and Reviews, 7(28), 619-626.

Conderman, G. y Hedin, L. (2015). Differentiating Instruction in Co-Taught Classrooms for Students with Emotional/Behaviour Difficulties. Emotional \& Behavioural Difficulties, 20(4), 349-361. DOI: http://doi.org/10.1080/13632752.2014.976918.

Cutri, R. y Whiting, D. F. (2015). The Emotional Work of Discomfort and Vulnerability in Multicultural Teacher Education. Teachers and Teaching: Theory and Practice, 21(8), 1010-1025. DOI: http://doi.org/10.1080/13540602.2015.1005869.

Daghan, G. y Akkoyunlu, B. (2014). A Qualitative Study about Performance Based Assesment Methods Used in Information Technologies Lesson. Educational Sciences: Theory and Practice, 14(1), 333-338.

Dolan, A. M. (2012). Reforming Teacher Education in the Context of Lifelong Learning: The Case of the BEd Degree Programme in Ireland. European Journal of Teacher Education, 35(4), 463-479. DOI: http://doi.org/10.1080/02619768.2012.696190.

Decker, S. L. y Roberts, A. M. (2015). Specific Cognitive Predictors of Early Math Problem Solving. Psychology in the Schools, 52(5), 477-488. DOI: http://doi. org/10.1002/pits.21837.

Ewing, D. L., Monsen, J. J. y Kwoka, M. (2014). Behavioural and Emotional WellBeing of Children Following Non-Directive Play with School Staff. Educational Psychology in Practice, 30(2), 192-203. DOI: http://doi.org/10.1080/02667363. 2014.907128.

Flecha, R. y Villarejo, B. (2015). Pedagogía Crítica: Un Acercamiento al Derecho Real de la Educación. Revista Internacional de Educación para la Justicia Social (RIEJS), 4(2), 87-100. DOI: http://doi.org/10.15366/riejs2015.4.2.

Ford-Connors, E., Dougherty, S., Robertson, D. A. y Paratore, J. R. (2015). Mediating Complex Texts in the Upper Grades: Considering Motivation, Instructional Intensity, and Cognitive Challenge. Journal of Adolescent \& Adult Literacy, 58(8), 650659. DOI: http://doi.org/10.1002/jaal.418.

Habók, A. (2015). Learning to Learn in Years 1 and 2 of Hungarian Primary Schools. Education, 43(2), 153-163. DOI: http://doi.org/10.1080/03004279.2013.783875.

Hortigüela, D., Pérez-Pueyo, A. y Fernández Río, J. (2016). Influencia de las experiencias vivenciadas por el alumnado en el desempeño de futuras competencias docentes. Contextos Educativos. Revista de Educación, 19, 27-43. DOI: http://doi. org/10.18172/con.2742.

Lee, E. Mishna, F. y Brennenstuhl, S. (2010). How to Critically Evaluate Case Studies in Social Work. Research on Social Work Practice, 20(6), 682-689. DOI: http:// doi.org/10.1177/1049731509347864.

Lopez-Gómez, M. (2015). Nuevos frentes de actuación en educación para el desarrollo humano. E-DHC, Quaderns Electrònics sobre el Desenvolupament Humà $i$ la Cooperació, 4, 17-37.

López-Pastor, V. M. y Palacios, A. (2013). Haz lo que yo digo pero no lo que yo hago: sistemas de evaluación del alumnado en la formación inicial del profesorado. Revista de Educación, 361, 279-305. 
Mincu, M. E. (2015). Teacher Quality and School Improvement: What Is the Role of Research? Oxford Review of Education, 41(2), 253-269. DOI: http://doi.org/10.10 80/03054985.2015.1023013.

Mirzagitova, A. L. y Akhmetov, L. G. (2015). Self-Development of Pedagogical Competence of Future Teacher. International Education Studies, 8(3), 114-121.

Mosha, M. A. (2015). The Role of Teachers' Resource Centres in Teachers' Professional Development and Enhancing Primary Education in Zanzibar. Journal of Education and Practice, 6(8), 44-61.

Olsson, D. y Gericke, N. (2016). The Adolescent Dip in Students' Sustainability Consciousness-Implications for Education for Sustainable Development. Journal of Environmental Education, 47(1), 35-51. DOI: http://doi.org/10.1080/00958964.201 5.1075464 .

O'Sullivan, S. (2011). Applying the Scholarship of Teaching and Learning in an Irish Context: Mission Impossible? Teaching Sociology, 39(3), 303-319. DOI: http://doi. org/10.1177/0092055X11407287.

Petersen, A. (2012). Imagining the Possibilities: Qualitative Inquiry at the Intersections of Race, Gender, Disability, and Class. International Journal of Qualitative Studies in Education (QSE), 25(6), 801-818. DOI: http://doi.org/10.1080/09518398.2011.645513.

Saldaña, J. (2009). The Coding Manual for Qualitative Researchers. Thousand Oaks, CA: Sage.

Sönmez, D. (2015). Creating Meaningful Experiences for Pre-Service Teachers: Thoughts and Experiences on an Elective Course. International Journal of Progressive Education, 11(2), 29-38.

Strauss, A. y Corbin, J. (2002). Bases de la investigación cualitativa: Técnicas y procedimientos para desarrollar la teoría fundamentada. Colombia: Universidad de Antioquia.

Sucaromana, U. (2012). Contribution to Language Teaching and Learning: A Review of Emotional Intelligence. English Language Teaching, 5(9), 54-58.

Thompson, M. y Beymer, P. (2015). The Effects of Choice in the Classroom: Is There Too Little or Too Much Choice? Support for Learning, 30(2), 105-120. DOI: http:// doi.org/10.1111/1467-9604.12086.

Winans, A. (2012). Cultivating Critical Emotional Literacy: Cognitive and Contemplative Approaches to Engaging Difference. College English, 75(2), 150-170.

Yuan, R. y Lee, I. (2015). The Cognitive, Social and Emotional Processes of Teacher Identity Construction in a Pre-Service Teacher Education Programme. Research Papers in Education, 30(4), 469-491. DOI: http://doi.org/10.1080/02671522.201 4.932830 .

Zembylas, M. (2012). Critical Emotional Praxis for Reconciliation Education: Emerging Evidence and Pedagogical Implications. Irish Educational Studies, 31(1), 1933. DOI: http://doi.org/10.1080/03323315.2011.579480.

Zlatkin-Troitschanskaia, E., Blömeke, S. y Pant, H. (2015). Competency Research in Higher Education: Conceptual and Methodological Challenges and Perspectives for Future Interdisciplinary Research. Peabody Journal of Education, 90(4), 459464. DOI: http://doi.org/10.1080/0161956X.2015.1068055. 